\title{
REVIEW OF SOLAR NEUTRINO EXPERIMENTS
}

\author{
A. BELLERIVE \\ Ottawa-Carleton Institute for Physics, Department of Physics, \\ Carleton University, 1125 Colonel By Drive, Ottawa, K1S 5B6, Canada \\ E-mail: alain_bellerive@carleton.ca
}

\begin{abstract}
This paper reviews the constraints on the solar neutrino mixing parameters with data collected by the Homestake, SAGE, GALLEX, Kamiokande, SuperKamiokande, and SNO experiments. An emphasis will be given to the global solar neutrino analyses in terms of matter-enhanced oscillation of two active flavors. The results to-date, including both solar model dependent and independent measurements, indicate that electron neutrinos are changing to other active types on route to the Earth from the Sun. The total flux of solar neutrinos is found to be in very good agreement with solar model calculations. Future measurements will focus on greater accuracy for mixing parameters and on better sensitivity to low neutrino energies.
\end{abstract}

\section{Introduction}

The deficit of neutrinos detected coming from the Sun compared with our expectations based on laboratory measurements, known as the Solar Neutrino Problem, has remained one of the outstanding problems in basic physics for over thirty years. It appeared inescapable that either our understanding of the energy producing processes in the Sun is seriously defective, or neutrinos, some of the fundamental particles in the Standard Model, have important properties which have yet to be identified. It was indeed argued by some that we needed to change our ideas on how energy was produced in fusion reactions inside the Sun. Others suggested that the problem arose due to peculiar characteristics of neutrinos such as oscillations and matter effects. It is then useful to review the evolution of our understanding from the data collected by various solar neutrino experiments. For completeness, new results from the Sudbury Neutrino Observatory not presented at the conference are included in the discussion presented here.

\section{Solar Neutrinos}

Hans Bethe suggested that the energy in the Sun was produced by nuclear reactions that allow hydrogen to be transformed into helium. ${ }^{1}$ This nuclear time scale is about $10^{10}$ years and corresponds to the epoch over which a star evolves in the main sequence. The Sun evolves slowly by adjusting its temperature so that the average thermal energy of a nucleus is small compared to the Coulomb repulsion an ion feels from potential fusion partners. The large Coulomb repulsion slows the nuclear rate to an astronomically long
Table 1. Neutrino production from fusion reactions in the Sun. ${ }^{2}$ The total solar flux at the Earth is $6.5 \times 10^{10}$ neutrinos per $\mathrm{cm}^{2}$ and per second. The majority of the solar neutrinos come from the $p p$ chain (more than $91 \%$ ); while the ${ }^{7} B e, p e p$, and ${ }^{8} B$ chains correspond to about $7 \%, 0.2 \%$, and $0.008 \%$ of the total flux, respectively. The hep contribution is minuscule and mostly neglected.

\begin{tabular}{|c|c|c|}
\hline Reaction & Label & Flux $\left(\mathrm{cm}^{-2} \mathrm{~s}^{-1}\right)$ \\
\hline$p+p \rightarrow{ }^{2} \mathrm{H}+e^{+}+\nu_{e}$ & $p p$ & $5.95 \times 10^{10}$ \\
$p+e^{-}+p \rightarrow{ }^{2} \mathrm{H}+\nu_{e}$ & $p e p$ & $1.40 \times 10^{8}$ \\
${ }^{3} \mathrm{He}+p \rightarrow{ }^{4} \mathrm{He}+e^{+}+\nu_{e}$ & $h e p$ & $9.3 \times 10^{3}$ \\
${ }^{7} \mathrm{Be}+e^{-} \rightarrow{ }^{7} \mathrm{Li}+\nu_{e}$ & ${ }^{7} \mathrm{Be}$ & $4.77 \times 10^{9}$ \\
${ }^{8} \mathrm{~B} \rightarrow{ }^{8} \mathrm{Be} e^{*}+e^{+}+\nu_{e}$ & ${ }^{8} B$ & $5.05 \times 10^{6}$ \\
\hline
\end{tabular}

time scale. Hence the rate for nuclear reactions in the solar interior is dominated by Coulomb barriers. Through the reactions listed in Table 1, four protons combine to form the helium nucleus containing two protons and two neutrons. As the protons only fuse to make helium under the very high density, high temperature conditions present at the centre of the Sun, it is virtually impossible to reproduce these conditions in the laboratory and hence study directly the nuclear solar fusion hypothesis. Considering also that it takes a photon about ten thousand years to reach the surface of the Sun from the core, investigation of the electromagnetic spectrum considering the latter time scale dilutes all clues about the origin and production mechanism of the photons.

However, somehow in the fusion process, two of the protons have to become neutrons. The only reactions that allow this to happen are caused by weak interactions responsible for nuclear beta decay and, each time a neutron is formed, there must be an associated electron neutrino produced. Neutrinos can travel directly from the core of the Sun to the Earth 


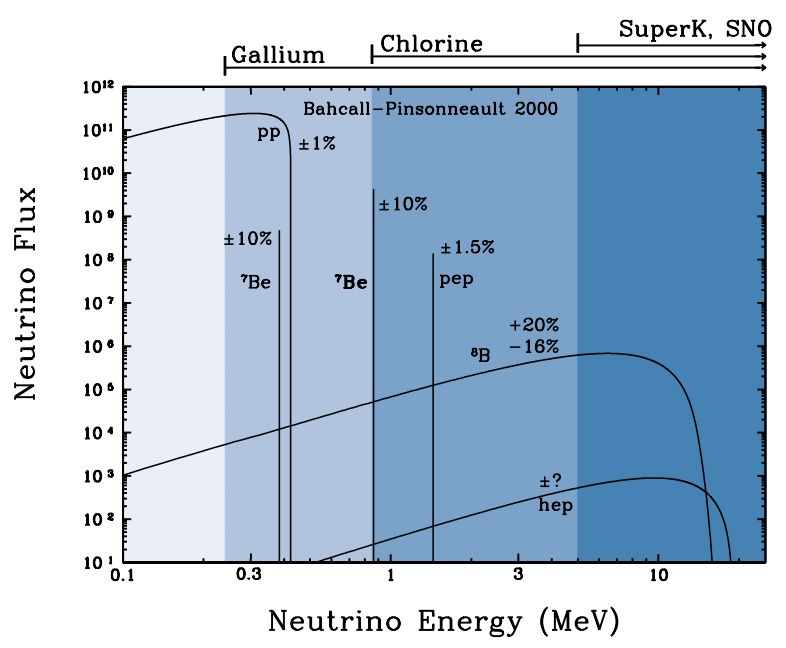

Figure 1. The solar neutrino spectra predicted by the SSM. The neutrino fluxes at one astronomical unit from continuum sources are given in units of $\mathrm{cm}^{-2} \mathrm{~s}^{-1} \mathrm{MeV}^{-1}$, and the line fluxes are given in $\mathrm{cm}^{-2} \mathrm{~s}^{-1}$. Courtesy of J.N. Bahcall from http://www.sns.ias.edu/ jnb/.

in a few minutes and hence provide a direct way to study processes by which protons form helium in the Sun. As early as 1949 Luis Alvarez proposed that the hypothesis of nuclear reactions powering the Sun could be tested by measuring the solar neutrino flux.

The number of neutrinos that we should expect is equal to twice the ratio of the energy received at the Earth in the form of sunshine, to the energy released when the four protons produce helium. The resulting number is huge: almost $10^{11}$ neutrinos pass through each square centimeter on Earth every second. Even with such numbers the detection proved a formidable challenge because of the very small scattering cross section of neutrinos on ordinary matter. The attraction of the measurement is nevertheless clear!

The detailed prediction of the electron neutrino flux created by the thermonuclear reactions in the interior of the Sun was performed by John Bahcall and his collaborators from the 1960's until now. Their calculations are referred to as the Standard Solar Model (SSM). In this paper, the BahcallPinsonneault calculations ${ }^{2}$ are used to compare experimental results and theoretical predictions. The solar neutrino spectra predicted by the SSM are shown in Fig. 1.

\section{Neutrino Oscillations}

It is known that neutrinos exist in different flavors corresponding to the three charged leptons: the electron, muon, and tau particles. If neutrinos have masses, flavor can mix in a charged-current interaction mediated by the $W$ boson. The neutrino emitted in a weak interaction is then a superposition of mass eigenstates

$$
\nu_{\ell}=\sum_{i=1}^{n} U_{\ell i}\left|\nu_{i}\right\rangle .
$$

The charged-current interactions in the leptonic sector are then described by the mixing matrix $U$

$$
U=\left(\begin{array}{cccc}
U_{e 1} & U_{e 2} & \cdots & U_{e n} \\
U_{\mu 1} & U_{\mu 2} & \cdots & U_{\mu n} \\
U_{\tau 1} & U_{\tau 2} & \cdots & U_{\tau n}
\end{array}\right) .
$$

Here the neutrino mass eigenstates are denoted by $\nu_{i}$ with $i=1,2, \cdots, n$, while the charged lepton flavor eigenstates are labeled $(e, \mu, \tau)$. In the case of three generations of neutrino, the matrix $U$ is called the Maki-Nakagawa-SakataPontecorvo (MNSP) matrix ${ }^{3}$ and appears analogous to the Cabibbo-Kobayashi-Maskawa (CKM) mixing matrix $^{4}$ in the quark sector. The MNSP can be factorized as

$$
U=U_{12} \times U_{23} \times U_{13}
$$

with

$$
\begin{gathered}
U_{12}=\left(\begin{array}{ccc}
c_{12} & s_{12} & 0 \\
-s_{12} & c_{12} & 0 \\
0 & 0 & 1
\end{array}\right), \\
U_{23}=\left(\begin{array}{ccc}
1 & 0 & 0 \\
0 & c_{23} & s_{23} \\
0-s_{23} & c_{23}
\end{array}\right), \\
U_{13}=\left(\begin{array}{ccc}
c_{13} & 0 & s_{13} e^{i \delta} \\
0 & 1 & 0 \\
-s_{13} e^{-i \delta} & 0 & c_{13}
\end{array}\right),
\end{gathered}
$$

where $c_{i j}=\cos \theta_{i j}, s_{i j}=\sin \theta_{i j}$, and $i, j$ denote the lepton generations. Possible CP-violation is naturally embedded in the phase $\delta$.

The leptonic mixing matrix naturally allows for flavor oscillations of the neutrinos. The most general form for solar neutrino oscillations can be simplified where only two neutrinos participate in the oscillation. The large neutrino flavor mixing between 
the second and third generation inferred from atmospheric neutrino data ${ }^{5}$ in conjunction with the absence of an oscillation signal in the $\mathrm{CHOOZ}$ reactor neutrino experiment ${ }^{6}$ requires a small component of one of the three mass eigenstates to the electron flavor eigenstate. Hence the survival probability for an electron neutrino to propagate in time can take the approximate form

$$
P_{e \alpha}=\delta_{e \alpha}-\left(2 \delta_{e \alpha}-1\right) \sin ^{2} 2 \theta \sin ^{2}\left(1.27 \frac{\Delta m^{2} L}{E}\right) .
$$

The mixing angle is represented by $\theta, L$ is the distance between the production point of $\nu_{e}$ and the point of detection of $\nu_{\alpha}, E$ is the energy of the neutrino, and $\Delta m^{2} \equiv m_{2}^{2}-m_{1}^{2}$ is the difference in the squares of the masses of the two states $\nu_{2}$ and $\nu_{1}$ which are mixing. The function $\delta_{e \alpha}$ is the usual Kronecker delta. The numerical constant 1.27 is valid for $L$ in meters, $E$ in $\mathrm{MeV}$, and $\Delta m^{2}$ in $\mathrm{eV}^{2}$. Consequently, the electron neutrino of energy $E$ produced in a weak interaction inside the Sun can then be described in vacuum by the Hamiltonian

$$
H=\left(\begin{array}{cc}
-\frac{\Delta m^{2}}{4 E} \cos 2 \theta & \frac{\Delta m^{2}}{4 E_{2}} \sin 2 \theta \\
\frac{\Delta m^{2}}{4 E} \sin 2 \theta & \frac{\Delta m^{2}}{4 E} \cos 2 \theta
\end{array}\right),
$$

such that

$$
i \frac{d}{d t}\left(\begin{array}{c}
\nu_{e} \\
\nu_{\beta}
\end{array}\right)=\frac{1}{2} H\left(\begin{array}{c}
\nu_{e} \\
\nu_{\beta}
\end{array}\right) .
$$

The notation $\beta=\mu \tau$ is often used to represent the other type of flavor present in the solar flux. In the context of the Solar Neutrino Problem, this suggests the investigation of (i) the disappearance of pure electron neutrinos produced in nuclear reactions when they reach the Earth, or (ii) the appearance of neutrinos of another flavor in the solar beam. The Earth-Sun distance is set by the planetary equations of Kepler. The energy of the neutrino depends on the type of nuclear reaction (c.f. Table 1) which produced the electron neutrino. By studying the time evolution of the solar neutrinos, all the physics is then embedded in the parameters $\theta$ and $\Delta m^{2}$. The full parameter space is covered with $\Delta m^{2} \geq 0$ and $0 \leq \theta \leq \frac{\pi}{2}$, or $0 \leq \theta \leq \frac{\pi}{4}$ and either sign for $\Delta m^{2}$. Note that the survival probability of Eq. (7) is invariant under the transformations $\Delta m^{2} \rightarrow-\Delta m^{2}$ and $\theta \rightarrow \frac{\pi}{2}-\theta$. These transformations redefine the mass eigenstates by $\nu_{1} \leftrightarrow \nu_{2}$. This situation implies that there is a two-fold discrete ambiguity in the interpretation of $P_{e \alpha}$ in the two-neutrino oscillation scheme: the two different sets of physical parameters $\left(\Delta m^{2}, \theta\right)$ and $\left(\Delta m^{2}, \frac{\pi}{2}-\theta\right)$ give the same transition probability in vacuum.

The observation of vacuum oscillations does not determine whether $\nu_{1}$ or $\nu_{2}$ is heavier. Hence, the measurement of $P_{e \alpha}$ in vacuum cannot differentiate whether the larger component of $\nu_{e}$ resides in the heavier or in the lighter neutrino mass eigenstate. Thus, a solution to the Solar Neutrino Problem implies the determination of one angle $\theta \equiv \theta_{12}$, one mass difference $\Delta m^{2} \equiv \Delta m_{12}^{2}$, and the sign of $\Delta m^{2}$. This corresponds to the extraction of the three MNSP elements: $U_{e 1}, U_{e 2}$, and $U_{e 3}$.

\section{Matter Effects}

As was realized by Mikheev and Smirnov, ${ }^{7}$ based on the formalism of Wolfenstein, ${ }^{8}$ the two-fold symmetry is lost when mixed neutrinos travel through regions of dense matter. In the Sun (or the Earth), neutrinos can undergo forward scattering with the particles in the medium. These interactions are, in general, flavor dependent. This possible modification of the oscillation pattern in matter is referred to as the Mikheev-Smirnov-Wolfenstein (MSW) effect. When neutrinos propagate through matter the Hamiltonian must be modified to include scattering of the electrons in the matter, both through neutralcurrent interactions (which affect all types of neutrinos equally and lead to no observable changes in the oscillation pattern) and through charged-current interactions, which at solar neutrino energies affect only the electron neutrinos.

The conditions that have to be satisfied for the MSW effect to occur involve the neutrino energy, $E$, and the local electron density, $N_{e}$. As the process is a resonant one, the effect can be strongly energy dependent. The evolution equation is still given by Eq. (9), but

$$
H=\left(\begin{array}{cc}
-\frac{\Delta m^{2}}{4 E} \cos 2 \theta+\sqrt{2} G_{F} N_{e} & \frac{\Delta m^{2}}{4 E} \sin 2 \theta \\
\frac{\Delta m^{2}}{4 E} \sin 2 \theta & \frac{\Delta m^{2}}{4 E} \cos 2 \theta
\end{array}\right),
$$

where $G_{F}$ is the Fermi coupling constant, while $\theta$ and $\Delta m^{2}$ are the usual mixing parameters. In the MSW oscillation scheme, the matter mixing angle term, $\sin ^{2} 2 \theta_{m}$, is related to the vacuum mixing an- 
gle, $\sin ^{2} 2 \theta$, by:

$$
\sin ^{2} 2 \theta_{m}=\frac{\sin ^{2} 2 \theta}{\left(w-\sin ^{2} 2 \theta\right)^{2}+\sin ^{2} 2 \theta},
$$

with

$$
w=-\sqrt{2} G_{F} N_{e} E / \Delta m^{2} .
$$

Thus, neutrinos created as electron-type in the centre of the Sun could emerge from the solar surface as mixed electron, muon, and tau neutrinos. The model also implies that, under certain conditions, muon or tau neutrinos striking the Earth could turn back into electron neutrinos leading to the intriguing possibility that the Sun might appear brighter to neutrino detectors at night than during the day.

\section{Chlorine Experiment}

The exploration of solar neutrinos started in the mid1960's with Ray Davis. ${ }^{9}$ It led to the first experiment that successfully detected neutrinos coming from the Sun. The experiment of Davis and his team was carried out deep underground in the Homestake mine in the US. The detector was based on a concept first proposed by Bruno Pontecorvo at Chalk River in 1946, in which neutrino reactions on chlorine are measured. Neutrinos striking chlorine can make an isotope of argon through the reaction

$$
\nu_{e}+{ }^{37} \mathrm{Cl} \rightarrow e^{-}+{ }^{37} \mathrm{Ar},
$$

with an energy threshold of $0.814 \mathrm{MeV}$. This reaction is rare and does not happen very often. In fact, about one atom of argon is produced each week in a tank containing 100,000 gallons of the dry-cleaning fluid, perchlorethylene. The challenge of this radiochemical experiment is to extract the few atoms of argon and count them by noting their decay back to chlorine which occurs with a half-life of 35 days. To carry out the low atom counting, the tank of dry-cleaning fluid is left for about a month and then purged with helium gas to sweep out the two or three atoms of argon. These atoms must then be separated from the helium by freezing them in a cold trap. They are then transferred to a low background counter where any decays are recorded over a period of several months. The first results were announced in 1968. The measurement clearly showed argon atoms produced by neutrinos, but the number was only one quarter of

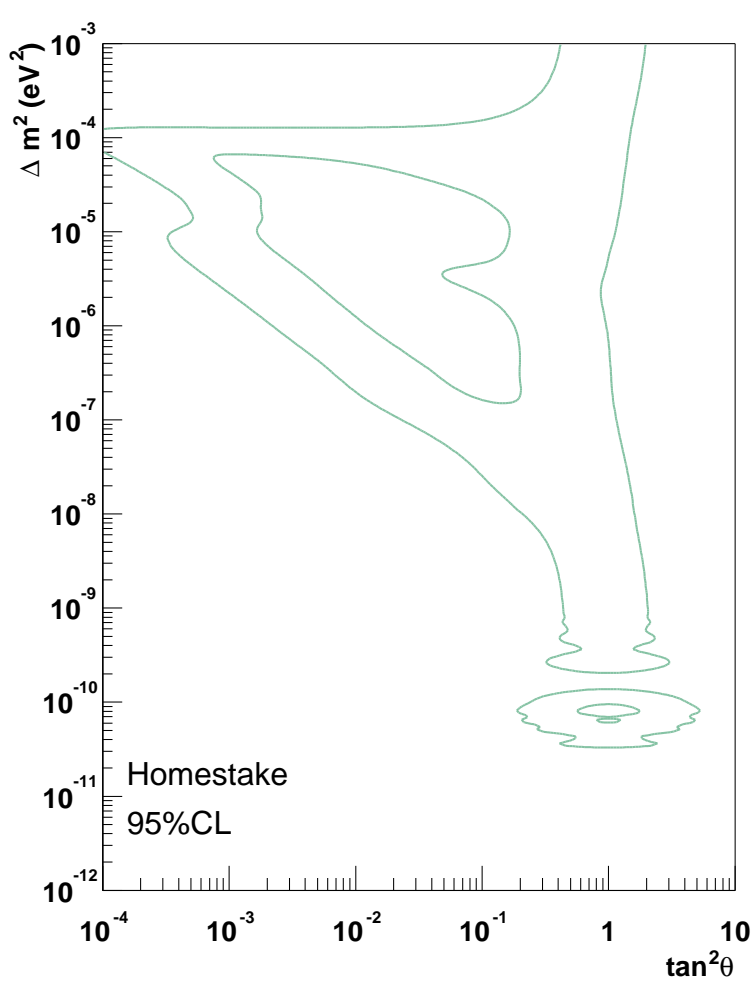

Figure 2. Mixing plane constraint provided by the chlorine experiment. The inside of the covariance regions is allowed at the $95 \%$ CL.

the predicted production rate. The chlorine experiment took data until 1995 and yielded ${ }^{10}$

$$
\Phi_{\mathrm{Cl}}=2.56 \pm 0.16 \pm 0.16 \mathrm{SNU},
$$

while the SSM predicted rate was

$$
\Phi_{\mathrm{Cl}}(\mathrm{SSM})=7.6_{-1.1}^{+1.3} \mathrm{SNU} .
$$

A SNU (Solar Neutrino Unit) is the product of the solar neutrino fluxes (measured or calculated) and the calculated cross sections. Hence one SNU equals one capture per second and per $10^{36}$ target atoms. The constraint on the oscillation parameters $\Delta m^{2}-\tan ^{2} \theta$ from the Homestake experiment is shown in Fig. 2. One uses $\tan ^{2} \theta$ instead of $\sin ^{2} 2 \theta$ so that $\tan ^{2} \theta<1$ corresponds to $m_{2}>m_{1}$ (normal hierarchy) and $\tan ^{2} \theta>1$ to $m_{2}<m_{1}$ (inverted hierarchy). The allowed region is obtained by comparing the measured and the calculated SSM solar neutrino fluxes (see Table 1). Ray Davis was awarded the 2002 Nobel Prize in physics for his pioneering work which provided the first evidence that the electron 
neutrino flux at the Earth, created by the thermonuclear reactions that power the Sun, is substantially less than would be predicted by the SSM.

\section{Gallium Experiments}

While the chlorine detector was mainly sensitive to the highest energy neutrinos (c.f. Fig. 1), two gallium experiments, one at the Baksan laboratory ${ }^{11}$ in Russia and one at the Gran Sasso laboratory ${ }^{12}$ in Italy, were set up to test the oscillation hypothesis at lower energy. The highest energy neutrinos and the production rate for these depends strongly on the solar central temperature; so a small change in the conditions was argued to give large changes in the predicted rates. On the other hand, the lower energy neutrino flux is expected to follow directly from the solar luminosity as discussed above. The motivation of the gallium experiments was then to disentangle which MSW neutrino oscillation scenario causes the Solar Neutrino Problem.

Like the ${ }^{37} \mathrm{Cl}$ detector, the gallium detectors could only detect electron type neutrinos because they looked for the reaction

$$
\nu_{e}+{ }^{71} G a \rightarrow e^{-}+{ }^{71} G e .
$$

The energy threshold of the ${ }^{71} G a$ detectors is $0.233 \mathrm{MeV}$ and hence allows the interaction of $p p$, ${ }^{7} \mathrm{Be},{ }^{8} \mathrm{~B}$, and pep neutrinos. The Russian-American group (SAGE) used a liquid metal target which contained 50 tons of gallium; while the European group (GALLEX/GNO) used 30 tons of natural gallium in an aqueous acid solution. Small proportional counters are used to count the germanium from the radiochemical target. The ${ }^{71} \mathrm{Ge}$ electron capture decay occurs with a half-life of 16.5 days. The Auger electrons and X-rays produce the typical L-peak and K-peak energy distribution. As a cross-check, both peaks are counted separately. A calibration with strong ${ }^{51} \mathrm{Cr}$ neutrino sources provides a nice verification for low atom chemical extraction and counting techniques. Improvements like new electronics, better radiation shielding, and improved calibration of counters are being pursued to continue regular data runs.

Both experiments found about half of the expected rate. The most recent results of $\mathrm{SAGE}^{13}$ were presented at LowNu 2003 and yield for the period 1990-2003:

$$
\Phi_{\mathrm{Ga}}=64.5_{-6.5}^{+6.8+3.7} \mathrm{SNU} \quad[\mathrm{L}-\mathrm{peak}],
$$

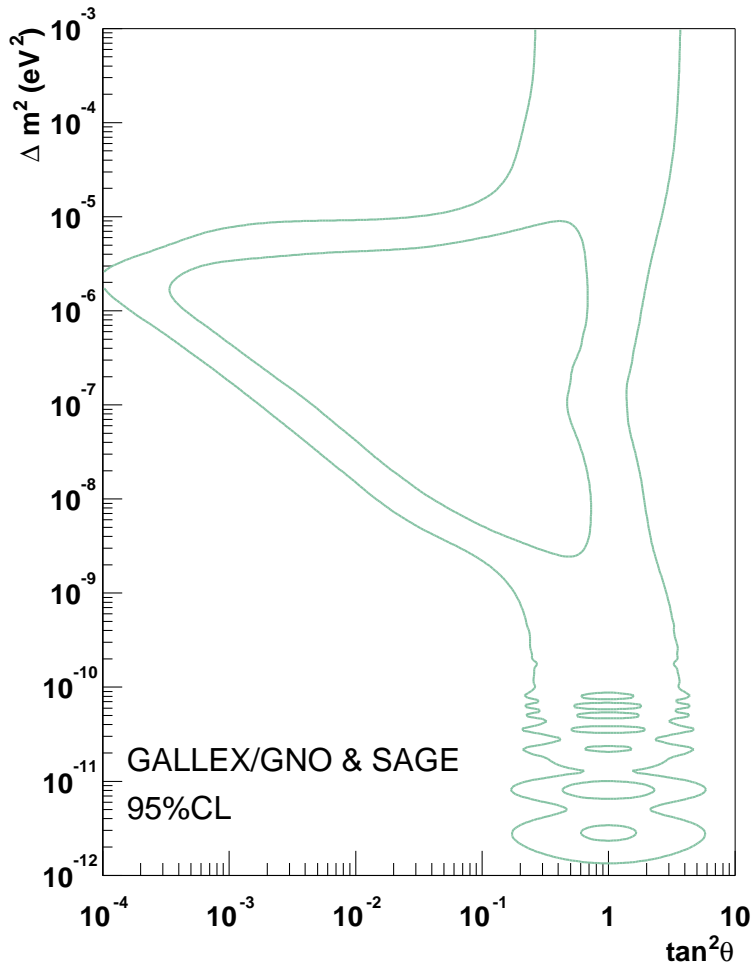

Figure 3. Constraint on the solar oscillation parameters from the gallium experiments. The inside of the covariance regions is allowed at the $95 \%$ CL. This exclusion plot uses the most recent results from SAGE and GALLEX/GNO.

$$
\begin{array}{lll}
\Phi_{\mathrm{Ga}}=73.3_{-5.7-3.2}^{+5.9} \mathrm{SNU} & {[\text { K-peak }]} \\
\Phi_{\mathrm{Ga}}=69.6_{-4.3}^{+4.4}+3.7 & \mathrm{SNU} & \text { [Overall] } .
\end{array}
$$

The GALLEX/GNO results for 1991-2002 were summarized at the Neutrino 2002 conference $^{14}$ and they were not updated for this symposium:

$$
\begin{array}{lll}
\Phi_{\mathrm{Ga}}=77.5 \pm 6.2 \pm 4.5 \mathrm{SNU} & {[\mathrm{GALLEX}]} \\
\Phi_{\mathrm{Ga}}=65.2 \pm 6.4 \pm 3.0 \mathrm{SNU} & {[\mathrm{GNO}],} \\
\Phi_{\mathrm{Ga}}=70.8 \pm 4.5 \pm 3.8 \mathrm{SNU} & {[\mathrm{GALLEX} / \mathrm{GNO}]}
\end{array}
$$

Yet again the data are incompatible with the SSM since the expected rate is

$$
\Phi_{\mathrm{Ga}}(\mathrm{SSM})=129_{-7}^{+9} \mathrm{SNU} .
$$

The constraint of the SAGE and GALLEX/GNO experiments on the MSW plane is shown in Fig. 3. The allowed region from the combination of the gallium results is obtained by comparing the measurements with the calculations of the SSM (see Table 1). 


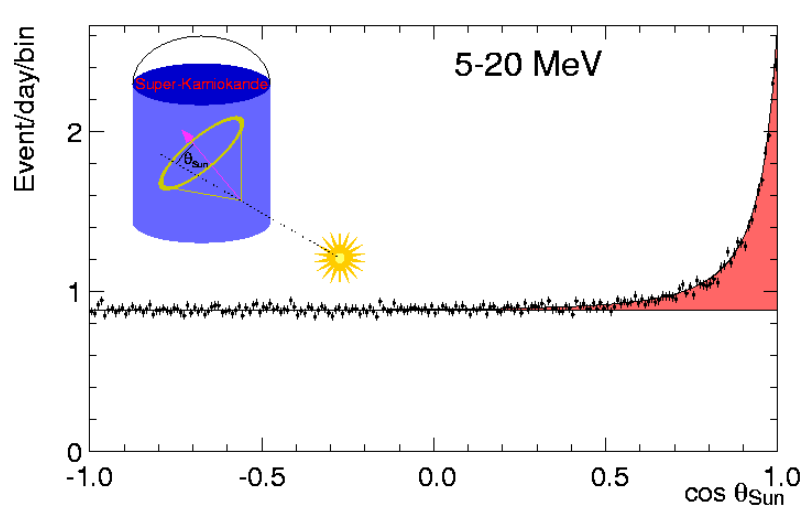

Figure 4. Angular distribution of solar neutrino candidates. SK data as of December 2002.

\section{Kamiokande and SuperKamiokande}

Following the first observations from the chlorine experiment the first priority was obviously an experimental confirmation of the solar-neutrino deficit. This was provided in 1987 by the Kamiokande water Čerenkov detector ${ }^{15}$ in Japan, which also saw a significant (but, interestingly enough, not an identical) suppression of the measured rate of neutrinos from the Sun. This great achievement was rewarded by the 2002 Nobel Prize in physics to Masatoshi Koshiba. The main advantage of the Kamiokande detector is the real-time nature of the neutrino interactions viewed in the active fiducial volume $(2,140$ tons of ultra-pure light water) by 948 photomultiplier tubes (PMT). The Kamiokande Collaboration demonstrated that the neutrinos are actually coming from the direction of the Sun by reconstructing the direction of flight of the incident neutrinos from the neutrino-electron scattering (ES) reaction $\nu_{x}+e^{-} \rightarrow$ $\nu_{x}+e^{-}$. Light water detectors are mainly sensitive to $\nu_{e}$, but also to $\nu_{\mu}$ and $\nu_{\tau}$, with a reduced cross section $\sigma\left(\nu_{\mu \tau} e^{-} \rightarrow \nu_{\mu \tau} e^{-}\right) \simeq 0.15 \times \sigma\left(\nu_{e} e^{-} \rightarrow \nu_{e} e^{-}\right)$.

The follow-up of the Kamiokande project is called the SuperKamiokande (SK) experiment. ${ }^{16}$ It was built to investigate in more detail the nature of atmospheric and solar neutrino oscillations. The SK detector is a huge, $40 \mathrm{~m}$ in diameter and $40 \mathrm{~m}$ high, circular cylinder filled with 50,000 tons of ultra-pure light water. The SK detector operated at an energy threshold of $5 \mathrm{MeV}$ and hence permitted the study of the ${ }^{8} B$ neutrinos. It is divided into an outer detector to veto incoming cosmic ray muons and to shield external low energy background; and an in-

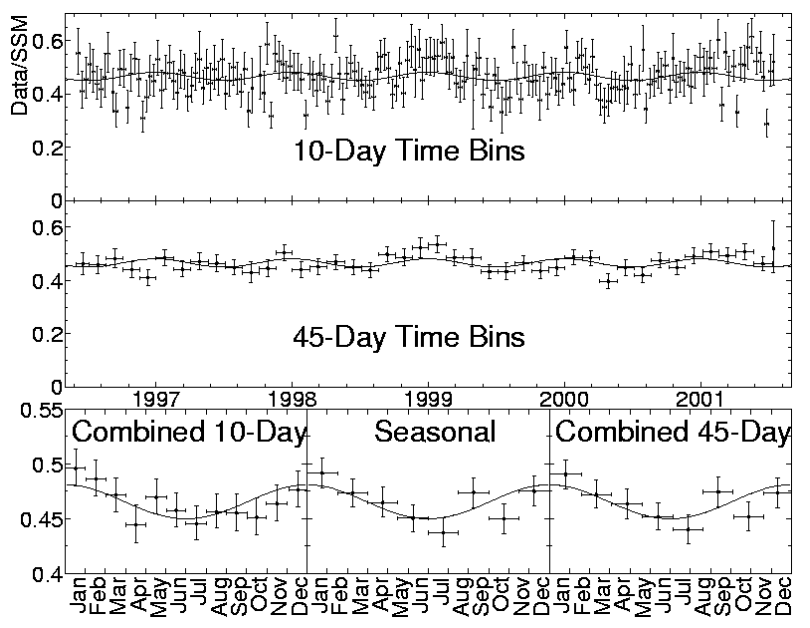

Figure 5. Time variation of the ES flux scaled by the SSM prediction. The curves represent the expected flux modulation due to the eccentricity of the Earth's orbit. SK data as of December 2002.

ner detector $(32,000$ tons, of which 22,500 tons is the active fiducial volume) viewed by 11,146 PMT. As in Kamiokande, solar neutrinos are observed by detecting Cerenkov photons emitted by the electrons resulting from ES events. The event rate was about 15 events per day (substantially larger than the rate in the radiochemical experiments).

The number of Cerenkov photons collected by the PMT can be calibrated as a measurement of the electron energy, while the position and times of the hit phototubes can be used to reconstruct the $\nu_{x}-e^{-}$interaction vertex and the electron direction. As mentioned before and as depicted in Fig. 4, the electron direction shows a strong peak pointing directly away from the Sun and enables discrimination against low-energy backgrounds in the detector arising from radioactive contaminants and spallation products produced by penetrating cosmic ray muons. ${ }^{17,18}$

The SK data allows measurements of the time dependence of the ES flux. It led to the measurement of the day/night rate asymmetry ${ }^{18}$

$$
A_{\mathrm{DN}}=2 \frac{\Phi_{\mathrm{D}}-\Phi_{\mathrm{N}}}{\Phi_{\mathrm{D}}+\Phi_{\mathrm{N}}}=-0.021 \pm 0.020_{-0.012}^{+0.013},
$$

and the precise determination of the ES neutrino flux $^{18}$

$$
\Phi_{\mathrm{ES}}=(2.35 \pm 0.02 \pm 0.08) \times 10^{6} \mathrm{~cm}^{-2} \mathrm{~s}^{-1} .
$$

The energy shape of the recoil electron agrees well, within experimental errors, with that predicted from 
the neutrino spectrum from the beta decay of ${ }^{8} B$. The measurement of the absolute flux, however, is about $46.5 \%$ of that predicted by the SSM.

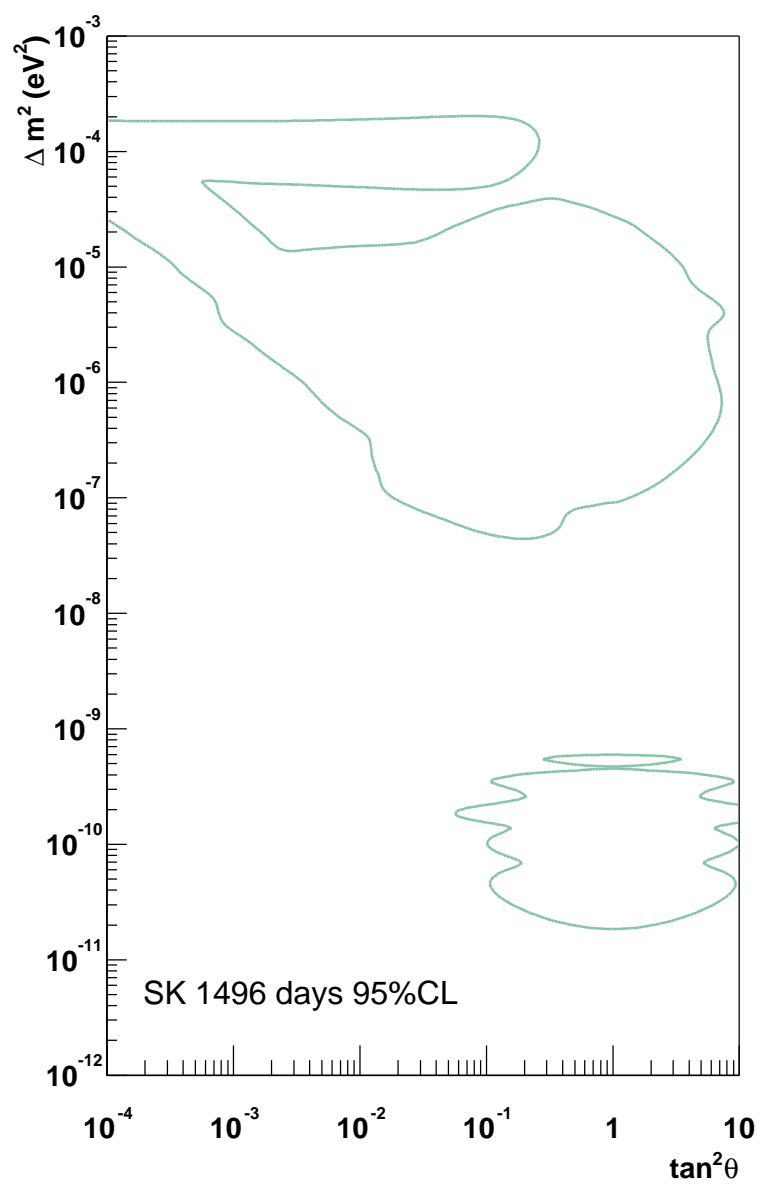

Figure 6. SSM flux independent excluded area using the SuperKamiokande zenith spectrum shape alone. The inside of the covariance regions is excluded at the $95 \%$ CL.

The real-time data of SK also provides the framework to study in detailed the shape of the zenith angle spectrum (i.e. the time and shape variation of the ES energy spectrum). The SK Collaboration looked at seasonal effects using 10 and 45 day bins. ${ }^{17,18}$ This is shown in Fig. 5. The time modulation of the measured ES flux is unfortunately consistent with the eccentricity of the Earth's orbit around the Sun. Therefore, beyond the rate difference between $\Phi_{\mathrm{ES}}$ of Eq. (19) and the SSM prediction, SK does not see any signature for oscillation from solar neutrinos. On the other hand, it is remarkable to visualize the complementarity of the radiochemical and SK experiments. Figure 6 shows the excluded regions of the mixing parameters from the SK Collaboration using the zenith spectrum shape alone. The lack of spectral distortion and daily variation breaks some degeneracy of the allowed regions of the chlorine and the gallium experiments.

Recently, SK looked for anti-neutrinos via the reaction $\bar{\nu}_{e}+p \rightarrow n+e^{+}$after removing solar neutrino candidates with the effective cuts $E>8 \mathrm{MeV}$ and $\cos \theta_{\text {sun }}<0.5$. From an accurate estimation of the intrinsic spallation background, which remains after the $\bar{\nu}_{e}$ selection criteria, they performed the first search for low energy $\bar{\nu}_{e}$ from the Sun (which is very relevant if neutrinos have a magnetic moment). In the absence of a signal, they reported an upper limit for the conversion probability to $\bar{\nu}_{e}$ of the ${ }^{8} B$ solar neutrinos. This conversion limit is $0.8 \%$ (90\% CL) of the SSM neutrino flux in the range of $8-20 \mathrm{MeV}^{19}$

\section{Sudbury Neutrino Observatory}

The Sudbury Neutrino Observatory (SNO) is a 1,000 ton heavy-water Čerenkov detector ${ }^{20}$ situated $2 \mathrm{~km}$ underground in INCO's Creighton mine in Canada. Another 7,000 tons of ultra-pure light water is used for support and shielding. The heavy water is in an acrylic vessel (12 $\mathrm{m}$ diameter and $5 \mathrm{~cm}$ thick) viewed by 9,456 PMT mounted on a geodesic structure $18 \mathrm{~m}$ in diameter; all contained within a polyurethanecoated barrel-shaped cavity ( $22 \mathrm{~m}$ diameter by $34 \mathrm{~m}$ high). The SNO detector has been filled with water since May 1999 and is moving toward the NeutralCurrent Detector (NCD) phase of its scientific program. The solar-neutrino detectors in operation prior to SNO were mainly sensitive to the electron neutrino type; while the use of heavy water by SNO allows the flux of all three neutrino types to be measured. Electron neutrinos can interact through a charged-current interaction while all neutrinos can interact through a neutral-current reaction. The determination of these reaction rates is a critical measurement in determining if neutrinos oscillate in transit between the core of the Sun and their observation on Earth.

Neutrinos from ${ }^{8} B$ decay in the Sun are observed in SNO from Čerenkov processes following these reactions:

- Charged-current (CC) reaction, specific to elec- 
tron neutrinos:

$$
d+\nu_{e} \rightarrow p+p+e^{-} .
$$

This reaction has a $\mathrm{Q}$ value of $1.4 \mathrm{MeV}$ and the electron energy is strongly correlated with the neutrino energy, providing potential sensitivity to spectral distortions.

- Neutral-current (NC) reaction, equally sensitive to all non-sterile neutrino types $(x=e, \mu, \tau)$ :

$$
\nu_{x}+d \rightarrow n+p+\nu_{x}
$$

This reaction has a threshold of $2.2 \mathrm{MeV}$ and is observed through the detection of neutrons by three different techniques in separate phases of the experiment.

- Elastic-scattering (ES) reaction:

$$
\nu_{x}+e^{-} \rightarrow \nu_{x}+e^{-} .
$$

This reaction has a substantially lower cross section than the other two and as mentioned before is predominantly sensitive to electron neutrinos.

The relations

$$
\begin{gathered}
\Phi_{\mathrm{CC}}=\phi_{e}, \\
\Phi_{\mathrm{ES}}=\phi_{e}+0.15 \phi_{\mu \tau}, \\
\Phi_{\mathrm{NC}}=\phi_{e}+\phi_{\mu \tau},
\end{gathered}
$$

give SNO the status of an appearance experiment. The SNO experimental plan calls for three phases of about two years each wherein different techniques will be employed for the detection of neutrons from the NC reaction. During the first phase, with pure heavy water, neutrons were observed through the Čerenkov light produced when neutrons were captured on deuterium, producing $6.25 \mathrm{MeV}$ gammas. In this phase, the capture probability for such neutrons was about $25 \%$ and the Čerenkov light is relatively close to the threshold of about $5 \mathrm{MeV}$ for the electron energy, imposed by radioactivity in the detector. For the second phase, about 2 tons of $\mathrm{NaCl}$ was added to the heavy water and neutron detection was enhanced through capture on $\mathrm{Cl}$, with about 8.6 MeV gamma energy release and about $83 \%$ capture effciency. Here, results from the pure $\mathrm{D}_{2} \mathrm{O}$ and salt phases will be reported. For the third phase, the salt will be removed and an array of ${ }^{3} \mathrm{He}$-filled

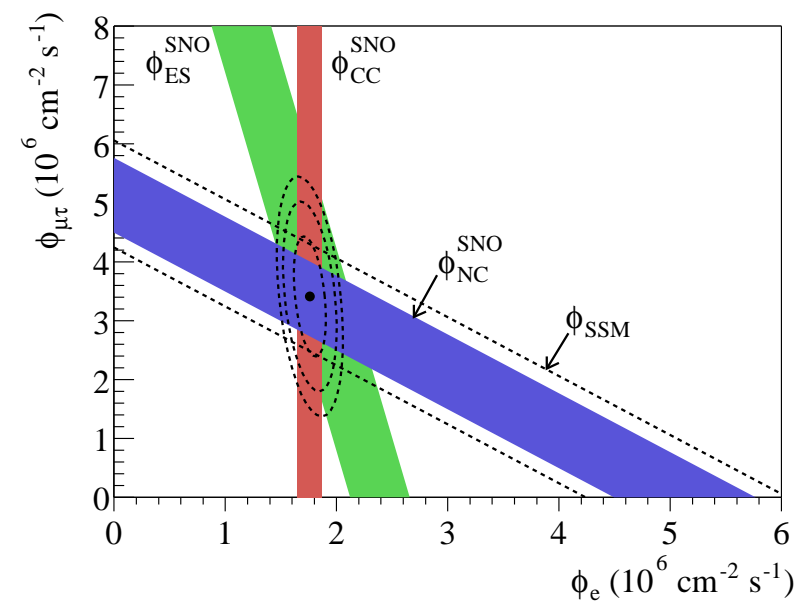

Figure 7. Flux of ${ }^{8} B$ solar neutrinos which are of $\mu$ or $\tau$ flavor versus the flux of electron neutrinos deduced from the three neutrino reactions in SNO. The diagonal bands show the total ${ }^{8} B$ flux as predicted by the SSM (dashed lines) and that measured with the $\mathrm{NC}$ reaction in SNO (solid band). The intercepts of these bands with the axes represent the $\pm 1 \sigma$ errors. The bands intersect at the fit values for $\phi_{e}$ and $\phi_{\mu \tau}$, indicating that the combined flux results are consistent with neutrino flavor transformation at the $5.3 \sigma$ level.

proportional counters will be installed to provide direct detection of neutrons with a capture efficiency of about $45 \%$.

During the pure $\mathrm{D}_{2} \mathrm{O}$ phase of the experiment, the signal was extracted with a statistical analysis technique based on the direction, $\cos \theta_{\text {sun }}$, the position, $R$, and the kinetic energy, $T_{e}$, of the event assuming the SSM energy spectrum shape. ${ }^{21}$ The final selection criteria were $T_{e} \geq 5 \mathrm{MeV}$ and $R \leq 550 \mathrm{~cm}$. An extended maximum-likelihood fit yields ${ }^{22}$

$$
\begin{aligned}
\Phi_{\mathrm{CC}} & =1.76_{-0.05}^{+0.06-0.09} \times 10^{6} \mathrm{~cm}^{-2} \mathrm{~s}^{-1}, \\
\Phi_{\mathrm{ES}} & =2.39_{-0.23}^{+0.24}{ }_{-0.12}^{+0.12} \times 10^{6} \mathrm{~cm}^{-2} \mathrm{~s}^{-1}, \\
\Phi_{\mathrm{NC}} & =5.09_{-0.43}^{+0.44+0.46} \times 1{ }_{-0.43}^{+0.46} \mathrm{~cm}^{-2} \mathrm{~s}^{-1} .
\end{aligned}
$$

The excess of the NC flux over the CC and ES fluxes implies neutrino flavor transformation. There is also a very nice agreement between the SNO NC flux and the total ${ }^{8} B$ flux of $5.05_{-0.81}^{+1.01} \times 10^{6} \mathrm{~cm}^{-2} \mathrm{~s}^{-1}$ predicted by the SSM. The simple change of variables in Eq. (20) resolves the data directly into electron and non-electron components ${ }^{22}$

$$
\begin{aligned}
\phi_{e} & =1.76_{-0.05}^{+0.06}{ }_{-0.09}^{+0.09} \times 10^{6} \mathrm{~cm}^{-2} \mathrm{~s}^{-1}, \\
\phi_{\mu \tau} & =3.41_{-0.45}^{+0.45}+0.45 \times 10^{6} \mathrm{~cm}^{-2} \mathrm{~s}^{-1} .
\end{aligned}
$$

As depicted in Fig. 7, where the error ellipses represent the $68 \%, 95 \%$, and $99 \%$ joint probability con- 

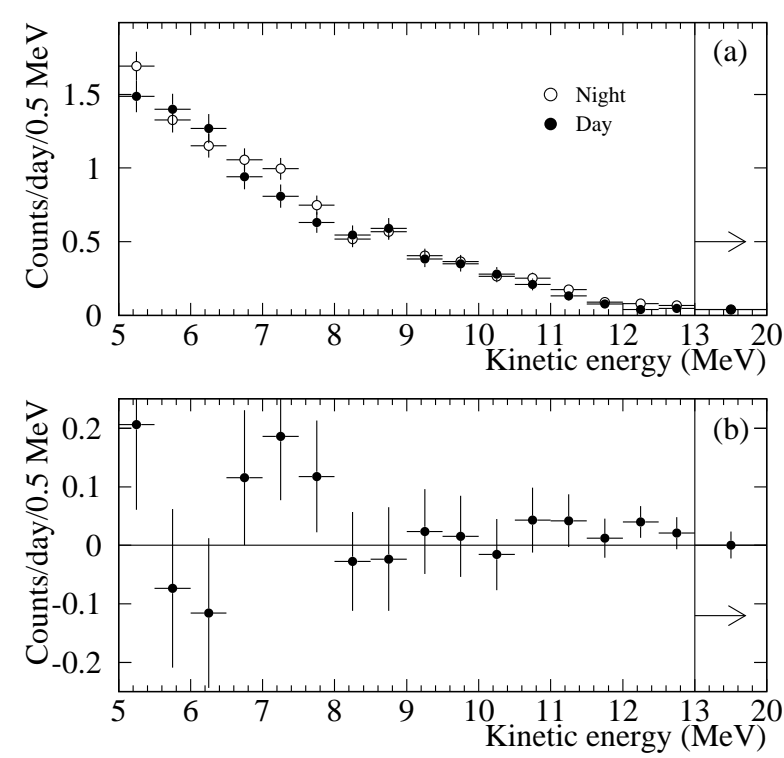

Figure 8. SNO (a) energy spectra for day and night. All signals and backgrounds contribute. The final bin extends from 13.0 to $20.0 \mathrm{MeV}$. (b) Difference, night - day, between the spectra. The day rate was $9.23 \pm 0.27$ events/day, and the night rate was $9.79 \pm 0.24$ events/day.

tours for $\phi_{e}$ and $\phi_{\mu \tau}$, there is clear evidence of solar neutrino flavor transformation at 5.3 standard deviations.

Allowing a time variation of the total flux of solar neutrino leads in SNO to day/night measurements which are sensitive to neutrino type $\mathrm{e}^{23}$

$$
\begin{array}{r}
A_{\mathrm{DN}}(\text { total })=\left(-24.2 \pm 16.1_{-2.5}^{+2.4}\right) \%, \\
A_{\mathrm{DN}}(e)=\left(12.8 \pm 6.2_{-1.4}^{+1.5}\right) \% .
\end{array}
$$

By enforcing no asymmetry in the total rate, i.e. $A_{\mathrm{DN}}($ total $)=0$, the day $/$ night asymmetry for the electron neutrino is ${ }^{23}$

$$
A_{\mathrm{DN}}(e)=\left(7.0 \pm 4.9_{-1.2}^{+1.3}\right) \% .
$$

The day and night energy spectra for all accepted events are shown in Fig. 8. Backgrounds were subtracted separately for day and night as part of the signal extraction. No systematic has been identified, in either signal or background regions, that would suggest that the small difference between day and night is other than a statistical fluctuation.

Even if they were not ready for this conference, SNO published their first results of the salt phase ${ }^{24}$ shortly after. The measurements were made with dissolved $\mathrm{NaCl}$ in the heavy water to enhance the sensitivity and signature for neutral-current interactions. Neutron capture on ${ }^{35} \mathrm{Cl}$ typically produces multiple $\gamma$ rays while the $\mathrm{CC}$ and ES reactions produce single electrons. The greater isotropy of the Čerenkov light from neutron capture events relative to CC and ES events allows better statistical separation of the event types. More importantly, this separation allows a precise measurement of the NC flux to be made independently of assumptions about the CC and ES energy spectra. The degree of the Cerenkov light isotropy is determined from the pattern of PMT hits. To minimize the possibility of introducing biases, SNO performed a blind analysis procedure for the more model independent determination of the total active $\left(\nu_{x}\right)^{8} B$ solar neutrino flux. The salt analysis was performed on the new data set, statistically separating events into CC, NC, ES, and external-source neutrons using an extended maximum-likelihood fit based on the distributions of isotropy, cosine of the event direction relative to the vector from the Sun, $\cos \theta_{\text {sun }}$, and radius, $R$, within the detector. This analysis differs from the previous analyses of the pure $\mathrm{D}_{2} \mathrm{O}$ data ${ }^{22,23}$ since the spectral distributions of the ES and CC events are not constrained to the ${ }^{8} B$ shape, but are extracted from the data. Cerenkov event backgrounds from $\beta-\gamma$ decays were reduced with an effective electron kinetic energy threshold $T_{e} \geq 5.5 \mathrm{MeV}$ and a fiducial volume with radius $R \leq 550 \mathrm{~cm}$. The extended maximumlikelihood analysis gives the following ${ }^{8} B$ fluxes ${ }^{24}$

$$
\begin{aligned}
\Phi_{\mathrm{CC}} & =1.59_{-0.07}^{+0.08}+0.06 \times 10^{6} \mathrm{~cm}^{-2} \mathrm{~s}^{-1}, \\
\Phi_{\mathrm{ES}} & =2.21_{-0.26}^{+0.31} \pm 0.10 \times 10^{6} \mathrm{~cm}^{-2} \mathrm{~s}^{-1}, \\
\Phi_{\mathrm{NC}} & =5.21 \pm 0.27 \pm 0.38 \times 10^{6} \mathrm{~cm}^{-2} \mathrm{~s}^{-1} .
\end{aligned}
$$

These fluxes are in agreement with previous SNO measurements and the SSM. The ratio of the ${ }^{8} B$ flux measured with the $\mathrm{CC}$ and $\mathrm{NC}$ reactions then provides a strong signature of solar neutrino oscillations

$$
\frac{\Phi_{\mathrm{CC}}}{\Phi_{\mathrm{NC}}}=0.306 \pm 0.026 \pm 0.024 \text {. }
$$

The salt shape-unconstrained fluxes presented here combined with shape-constrained fluxes and day/night energy spectra from the pure $\mathrm{D}_{2} \mathrm{O}$ phase $^{22,23}$ place impressive constraints on the allowed neutrino flavor mixing parameters. Two-flavor active neutrino oscillation models predict the $\mathrm{CC}$, $\mathrm{NC}$, and $\mathrm{ES}$ rates in SNO. In the fit, the ratio $f_{B}$ of the total ${ }^{8} B$ flux to the SSM value is a free parameter 


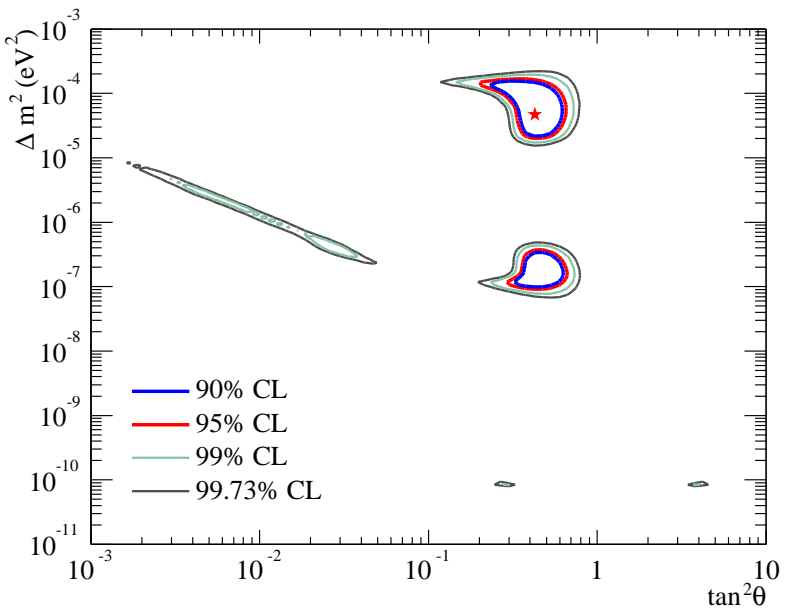

Figure 9. SNO-only neutrino oscillation contours, including pure $\mathrm{D}_{2} \mathrm{O}$ day/night spectra, salt CC, NC, ES fluxes, with ${ }^{8} B$ flux free and hep flux fixed. The best-fit point is $\Delta m^{2}=$ $4.7 \times 10^{-5}, \tan ^{2} \theta=0.43, f_{B}=1.03$, with $\chi^{2} /$ d.o.f. $=26.2 / 34$. The inside of the covariance regions is allowed.

Table 2. Overview of all the solar neutrino data. Only experimental errors are considered in the ratio of the data to the SSM predictions.

\begin{tabular}{|c|c|c|}
\hline Experiment & Reaction & Ratio data/SSM \\
\hline Chlorine & CC & $0.34 \pm 0.03$ \\
SAGE+GALLEX/GNO & CC & $0.55 \pm 0.03$ \\
SuperKamiokande & ES & $0.47 \pm 0.02$ \\
SNO & CC & $0.35 \pm 0.02$ \\
SNO & ES & $0.47 \pm 0.05$ \\
SNO & NC & $1.01 \pm 0.13$ \\
\hline
\end{tabular}

together with the mixing parameters. A combined $\chi^{2}$ fit to SNO $\mathrm{D}_{2} \mathrm{O}$ and salt data alone yields the allowed regions in $\Delta m^{2}$ and $\tan ^{2} \theta$ shown in Fig. 9.

\section{Global Fits}

This section summarizes the solar neutrino data in a global analysis of all experiments. Table 2 shows the experimental results compared with the SSM prediction. The data indeed suggest an energy dependence due to the different energy thresholds of the different detection techniques.

The global analysis presented here includes the chlorine results, ${ }^{10}$ the updated gallium flux measurements, ${ }^{13,14}$ the SK zenith spectra, ${ }^{17,18}$ and the $\mathrm{D}_{2} \mathrm{O}$ and salt results from SNO. ${ }^{22,23,24}$ The free parameters in the global fit are the $\operatorname{total}^{8} B$ flux, the difference of the squared mass $\Delta m^{2}$, and the mixing angle $\theta$. The higher energy hep flux is fixed at

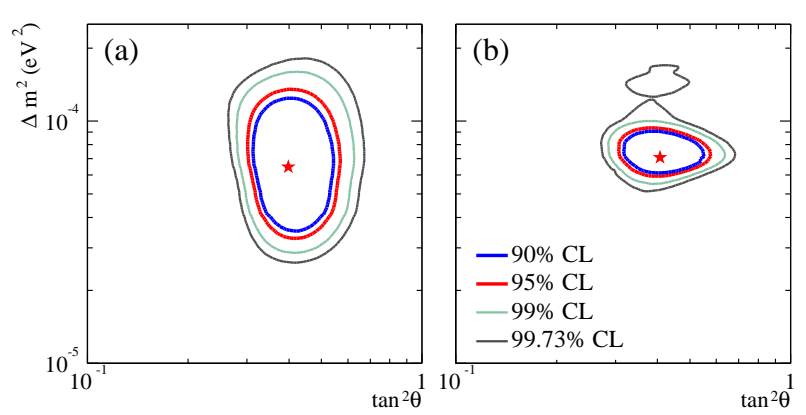

Figure 10. Allowed region of the MSW plane determined by a $\chi^{2}$ fit to (a) the chlorine, gallium, SK, and SNO experiments. The best-fit point is $\Delta m^{2}=6.5 \times 10^{-5}, \tan ^{2} \theta=0.40, f_{B}=$ 1.04, with $\chi^{2} /$ d.o.f. $=70.2 / 81$. (b) Solar global + KamLAND. The best-fit point is $\Delta m^{2}=7.1 \times 10^{-5}, \tan ^{2} \theta=0.41, f_{B}=$ 1.02. In the MSW analyses, the ratio $\left(f_{B}\right)$ of the total ${ }^{8} B$ flux to the SSM value is a free parameter, while the total hep flux is fixed to the SSM prediction. The inside of the covariance contours is the allowed region.

$9.3 \times 10^{3} \mathrm{~cm}^{-2} \mathrm{~s}^{-1}$. Contours are generated in $\Delta m^{2}$ and $\tan ^{2} \theta$ for $\Delta \chi^{2}=4.61(90 \% \mathrm{CL}), 5.99(95 \% \mathrm{CL})$, 9.21 (99\% CL), and 11.83 (99.73\% CL). As presented in Fig. 10(a), the combined results of all solar neutrino experiments can be used to determine a unique region of the MSW plane. In this global fit of all solar neutrino data, the allowed regions in parameter space shrink considerably and the Large Mixing Angle (LMA) region is selected.

A global analysis including the KamLAND reactor anti-neutrino results ${ }^{25}$ shrinks the allowed region further, with a best-fit point of $\Delta m^{2}=7.1_{-0.6}^{+1.2} \times$ $10^{-5} \mathrm{eV}^{2}$ and $\theta=32.5_{-2.3}^{+2.4}$ degrees, where the errors reflect $1 \sigma$ constraints on the 2 -dimensional region. This is summarized in Fig. 10(b). With the new SNO measurements the allowed region is constrained to only the lower band of LMA at > 99\% CL. The best-fit point with one dimensional projection of the uncertainties in the individual parameters (marginalized uncertainties) has $\Delta m^{2}=7.1_{-0.3}^{+1.0} \times 10^{-5} \mathrm{eV}^{2}$ and $\theta=32.5_{-1.6}^{+1.7}$ degrees. This disfavors maximal mixing $\left(\tan ^{2} \theta=1\right)$ at a level equivalent to 5.4 standard deviations.

\section{Future}

The upcoming generation of real-time low energy solar neutrino experiments will tackle new frontiers and allow the direct study of the mono-energetic ${ }^{7} \mathrm{Be}$ solar neutrinos. The goal of the Borexino and Kam- 
LAND experiments is in fact to measure the timedependence of the neutrino-electron scattering rate from neutrinos produced by electron capture on ${ }^{7} \mathrm{Be}$ in the Sun. The produced ES electrons are below threshold for production of Cerenkov light and thus liquid scintillator is used as detector target for enhancing the detection capability. The challenge at such a low threshold is the unprecedented low level of radioactivity required; namely less then $10^{-16} \mathrm{~g} / \mathrm{g}$ of ${ }^{238} \mathrm{U}$ and ${ }^{232} \mathrm{Th}$ and less than one part in $10^{18}$ of ${ }^{14} C$. One of the dominant backgrounds to be removed is radioactive Krypton $\left({ }^{85} K r\right)$. The major interest in the results for the next generation of low energy neutrino experiments arises when one compares the total ${ }^{7} \mathrm{Be}$ rate to the day/night rate asymmetry, $A_{\mathrm{DN}}$. This will give an excellent independent discrimination between different MSW solutions. It will therefore over-constrain the $\Delta m^{2}-\tan ^{2} \theta$ plane in a global analysis of all the solar neutrinos experiments.

The Borexino detector ${ }^{26}$ is located in Hall $\mathrm{C}$ of the Gran Sasso Laboratory. The time for the start of data taking is unclear due to an environmental problem encountered in 2002. Borexino is a 300 ton liquid-scintillator based detector with 100 tons of active fiducial mass in a $8.3 \mathrm{~m}$ diameter spherical nylon bag surrounded by a 2.6 meter thick spherical shell filled with buffer oil. The liquid scintillator and buffer liquid are viewed by 2,240 PMT which are mounted inside a $13.5 \mathrm{~m}$ diameter stainless steel tank; which is in turn surrounded by a $18 \mathrm{~m}$ spherical tank filled with ultra-pure light water to act as a radiation shield. The expected energy threshold is about $800 \mathrm{keV}$.

The KamLAND detector is located in the cavity used for the original Kamiokande experiment. The primary goal of KamLAND ${ }^{25}$ is to investigate the oscillation of $\bar{\nu}_{e}$ emitted from distant nuclear reactors. ${ }^{27}$ The investigation of ${ }^{7} \mathrm{Be}$ neutrinos comes for free in a later stage when their scientific program will shift from a coincidence experiment to an ES low energy experiment. The radioactive background remains of course the main concern for solar neutrino physics. KamLAND hosts 1,000 tons of liquid scintillator contained in a $13 \mathrm{~m}$ diameter spherical balloon, which is in turn surrounded by a $18 \mathrm{~m}$ spherical tank with 1,879 inward facing phototubes. The space between the balloon and the tank is filled with buffer oil and the containment tank is immersed in a 3,200 ton water Čerenkov detector instrumented with 225 PMT.

The other important next generation of real-time solar neutrino experiments should detect the fundamental $p p$ neutrinos, which constitute about $91 \%$ of the total neutrino flux predicted by the SSM. Unfortunately, there are no approved $p p$ solar neutrino experiments at the present time, although there are a number of promising proposals under development and $\mathrm{R} \& \mathrm{D}$ is ongoing.

\section{Summary}

Solar neutrino oscillation is clearly established by the combination of the results from the chlorine, gallium, SK, and SNO experiments. The real-time data of SK and SNO do not show large energy distortion nor time-like asymmetry. SNO provided the first direct evidence of flavor conversion of solar electron neutrinos by comparing the $\mathrm{CC}$ and $\mathrm{NC}$ rates. Matter effects explain the energy dependence of solar oscillation, and Large Mixing Angle (LMA) solutions are favored.

The global analysis of the solar neutrino detectors and reactor neutrino results yields $\Delta m^{2}=$ $7.1_{-0.3}^{+1.0} \times 10^{-5} \mathrm{eV}^{2}$ and $\theta=32.5_{-1.6}^{+1.7}$ degrees. Maximal mixing is rejected at the equivalent of 5.4 standard deviations and confirms the region $\tan ^{2} \theta<1$, which corresponds to the normal mass hierarchy of $m_{2}>m_{1}$ (i.e. $\Delta m^{2}>0$ ).

Solar neutrino data demonstrates that neutrinos have mass and that the minimal Standard Model is incomplete. Unlike the quark sector where the CKM mixing angles are small, the lepton sector exhibits large mixing. The neutrino masses and mixing may play significant roles in determining structure formation in the early universe as well as supernovae dynamics and the creation of matter. The coming decade will be exciting for neutrino physics helping to delineate the New Standard Model that will include neutrino masses and oscillations. This will lead to precision measurements of the leptonic mixing matrix, determination of neutrino masses, and investigation of $\mathrm{CP}$ and $\mathrm{CPT}$ properties in the lepton sector. After 30 years of hard labor from the nuclear and particle physics community, the Solar Neutrino Problem is now becoming an industry for precise measurements of neutrino oscillation parameters with the next generation of solar neutrino and long baseline neutrino experiments. 


\section{Acknowledgments}

This article builds upon the careful and detailed work of many people. Special thanks to E. Bellotti, M. Boulay, J. Formaggio, V. Gavrin, K. Graham, K. Heeger, R. Hemingway, A. Ianni, A. Marino, M. Nakahata, A. Poon, Y. Takeuchi, and J. Wilkerson. This research has been financially supported in Canada by the Natural Sciences and Engineering Research Council (NSERC), the Canada Research Chair (CRC) Program, and the Canadian Foundation for Innovation (CFI). I am grateful to the SNO Collaboration for giving me the opportunity to contribute to the 2003 Lepton Photon Symposium.

\section{References}

1. H.A. Bethe, Phys. Rev. 55, 436 (1939).

2. J.N. Bahcall, H.M. Pinsonneault, and S. Basu, Astrophys. J. 555, 990 (2001).

3. Z. Maki, M. Nakagawa, S. Sakata, Prog. Theor. Phys. 28, 870 (1962); B. Pontecorvo, Sov. Phys. JETP 26, 984 (1968).

4. N. Cabibbo, Phys. Rev. Lett. 10, 531 (1963); M. Kobayashi, and T. Maskawa, Prog. Theor. Phys. 49, 652 (1973).

5. Y. Fukuda et al., Phys. Rev. Lett. 81, 1562 (1998).

6. M. Apollonio, Phys. Lett. B 466, 415 (1999).

7. S.P. Mikheyev and A.Yu. Smirnov, Sov. J. Nucl. Phys. 42, 913 (1985).
8. L. Wolfenstein, Phys. Rev. D 17, 2369 (1978).

9. R. Davis, Jr., Phys. Rev. Lett., 302 (1964).

10. B.T. Cleveland et al., Ap. J. 496, 505 (1998).

11. J.N. Abdurashitov et al., Phys. Rev. C 60 (1999).

12. W. Hampel et al., Phys. Lett. B 447, 127 (1999); M. Altmann et al., Phys. Lett. B 490, 16 (2000).

13. V. Gavrin, $4^{\text {th }}$ International Workshop on Low Energy and Solar Neutrinos, Paris, May 19-21, 2003.

14. T. Kirsten, $X^{\text {th }}$ Int. Conf. on Neutrino Physics and Astrophysics, Munich, May 25-30, 2002; Nucl. Phys. B (Proc. Suppl.) 118 (2003).

15. Y. Suzuki, Nucl. Phys. (Proc. Suppl.) 34, 54 (1995).

16. M. Nakahata et al., Nucl. Inst. Meth. A 421, (1999).

17. S. Fukuda et al., Phys. Rev. Lett. 86, 5651 (2001).

18. S. Fukuda et al., Phys. Lett. B 539, 179 (2002).

19. S. Fukuda et al., Phys. Rev. Lett. 90, 171302 (2003).

20. J. Boger et al., Nucl. Inst. Meth. A 449, 172 (2000).

21. C.E. Ortiz et al., Phys. Rev. Lett. 85, 2909 (2000).

22. Q.R. Ahmad et al., Phys. Rev. Lett. 89, 011301 (2002).

23. Q.R. Ahmad et al., Phys. Rev. Lett. 89, 011302 (2002).

24. Q.R. Ahmad et al., submitted to Phys. Rev. Lett., Sept. 2003, nucl-ex/0309004.

25. K. Eguchi et al., Phys. Rev. Lett. 90, 021802 (2003).

26. G. Bellini, $X^{\text {th }}$ Int. Conf. on Neutrino Physics and Astrophysics, Munich, May 25-30, 2002; Nucl. Phys. B (Proc. Suppl.) 118 (2003); G. Alimonti et al., Astropart. Phys. 16, 205 (2002).

27. Kunio Inoue in these proceedings. 


\section{DISCUSSION}

Luc Declais (IPNL, France): Can you say more about the interest of real-time low energy $p p$ neutrino experiments for solar neutrinos, because GNO will measure the total amount of neutrinos above a given threshold with an accuracy of $7 \%$ ? So is more or less everything is known?

Alain Bellerive: When I first wrote the summary for future experiments, the last point was "The importance of $p p$ real-time neutrino experi- ments" and I changed it to "Real-time low energy neutrinos are the ultimate probe of the Sun and test of the Standard Solar Model". So you're right, at the moment if you can pin down the ${ }^{7} B e$ to really high accuracy, I think that it will provide a really good proof of matter effects in the context of the SSM. The $p p$ neutrinos are nevertheless an important piece of the puzzle since the majority of the neutrinos from the Sun are produced via the $p p$ reaction. 